\section{Operating on the Elderly}

Fifty years ago age itself was a contraindication to surgery, because the surgical and anaesthetic techniques of the time could not prevent an unacceptable mortality rate. Now age is no longer a bar to operation, but the chronic diseases, which are commoner with advancing age and increase the operative risk, must still be contended with. The physician and surgeon advising an elderly patient has to consider three aspects of the problem: firstly, the indications for operation and the benefit it will give the patient; secondly, the technical problems of the operation and the intraoperative complications; thirdly, the likelihood of postoperative complications and ways of preventing them.

Many elective operations are performed on young and middle-aged people because of the risk that the underlying disease will cause further symptoms. For example, most surgeons regard one attack of cholecystitis and the radiological detection of gallstones as indications for cholecystectomy. But such indications must be reconsidered when met with in a 70-year-old. How great are the chances of further attacks? Will they be severe? Do they really justify an operation with an appreciable mortality rate? No general guidance can be given on this problem. Each case must be assessed individually. One thing is certain: a little delay and extra reflection rarely does any harm and usually does a lot of good. It is wrong to hurry into any operation on an elderly patient if there are not clear and indisputable indications for it.

The tissues of the elderly heal as well as those of the young, and the surgeon need not generally expect the patient's age to make his technical performance more difficult. Wounds heal provided the nutrition of the patient is normal. Old people on a small income rarely show overt signs of malnutrition but many have a diet deficient in protein and vitamins. Consequently their wounds are slow to heal and lack resistance to infection. Time spent before an operation in restoring a patient's nutrition is never wasted. Some operations, particularly vascular ones, may be complicated by a sudden massive loss of blood. An elderly cardiovascular system cannot compensate as quickly and effectively as a young one, and unless the blood is replaced as soon as it is lost there may be profound, damaging hypotension. Operations which might place an excessive strain on the cardiovascular or respiratory systems must be planned and executed to keep intraoperative stress to a minimum. If stress is inevitable, the advisability of proceeding with the operation should be reconsidered.

Once the operation is over the real problems begin. The main cause of death is bronchopneumonia. ${ }^{1}$ This is related to three factors-pre-existing lung disease, the changes in the lungs during anaesthesia, and failure of the alveoli to expand and of the bronchioles to clear their secretions after the operation because of pain. During anaesthesia the anaesthetist can usually maintain oxygenation however diseased the lungs, but as soon as the patient breathes spontaneously ventilation often becomes inadequate and bronchial secretions accumulate in dependent areas. The mortality rate is greater if there is preoperative bronchitis and if the patient smokes. The lungs must be at their best before operation, and to ensure this may entail many days of preoperative assessment and preparation-chest radiographs, repeated spirometry, no smoking, breathing exercises, physiotherapy, antibiotics, and preoperative consultations between surgeon and anaesthetist. Proper preoperative preparation of the lungs is the main cause of the improved mortality rates of the past 10 years.
The elderly find coughing after an abdominal operation especially difficult. Analgesics given before the physiotherapy are a great help, as are supportive straps and binders. In the worst cases continuous or intermittent postoperative epidural anaesthesia can totally abolish pain and permit deep breathing and coughing. The diaphragm descends when the patient is upright, so the quicker he gets up the sooner the bases of the lungs will be fully expanded. ${ }^{2}$ The exercise of rising also causes tachypnoea and deeper breathing. Here the nurse and physiotherapist have to be encouraging but firm, for sympathetic acquiescence in a request to stay in bed another day can be harmful.

The only other preoperative disease likely to cause difficulties during and after the operation is recent myocardial ischaemia. The chances of myocardial infarction are high if the patient has suffered an infarct in the previous six months, and operation should be delayed if possible.

General care of pressure areas, micturition, and the bowels all play their part in ensuring a speedy recovery. A rapid recovery-that is the aim when operating on the elderly. The message of almost all the reports on this topic ${ }^{1-5}$ may be summed up in the phrase: Each day of preoperative preparation takes a week off the postoperative recovery.

1 Burnett, W., and McCaffrey, J., Surgery, Gynecology and Obstetrics, $1972,134,221$.

Browse, N. L., The Physiology and Pathology of Bed Rest. Springfield Illinois, C. Thomas, 1965.

${ }^{3}$ Brooks, B., Annals of Surgery, 1937, 105, 481.

4 Herron, P. W., Jesseph, J. E., and Harkins, H. N., Annals of Surgery, 1960, 152, 686

5 Ochsner, A., Geriatrics, 1967, No. 11, 22, 121.

\section{Retinal Vasculitis}

In 1880 Henry Eales, an ophthalmic surgeon in Birmingham, described a disease in which recurrent haemorrhages developed within the retina, particularly the more peripheral part, with extensions of the haemorrhage into the vitreous. ${ }^{1}$ It was seen in a group of males between the ages of 14 and 29 years, and it was regarded as a primary retinal disease. His paper proved to be a classic and has stood the test of time with remarkably few subsequent alterations, except for the knowledge that the disease is not confined exclusively to such a narrow age range and that it may occur also, though much less commonly, in the female. It is natural that the condition has become recognized widely as Eales's disease.

The aetiology of the disease has proved elusive, but it has been regarded generally as some form of inflammatory lesion of the retinal veins (periphlebitis) with an involvement occasionally of the retinal arteries (periarteritis), so that the term "perivasculitis" was suggested. But histological evidence of lesions in the walls of the retinal blood vessels rather than merely their surrounding connective tissues determined the more appropriate term "retinal vasculitis."2 Often Eales's disease occurs on its own, but occasionally it is associated with one of a variety of general disorders-multiple sclerosis, hemiplegia, paraplegia, transverse spinal cord lesions, thromboangiitis obliterans, tuberculosis, or brucellosis-and with an anterior or posterior uveitis. Unfortunately these associations do not help to establish a precise understanding of the aetiology, though it may be regarded as a non-specific tissue reaction to various antigens.

The brunt of the disease is borne by the veins in the periphery of the retina, so that any involvement of the 
central retina was considered at one time to be the result simply of a spread of the disease from the peripheral region. But sporadic reports have appeared which indicate a primary lesion of the central retinal vein within the optic nerve-head, and this central retinal vasculitis was described in detail by $\mathbf{T}$. K. Lyle and $\mathrm{K}$. Wybar. ${ }^{3}$ The condition, which is usually unilateral, shows several characteristic features: a considerable degree of oedema of the optic disc, which may extend into the macular area; a massive dilatation of the retinal veins extending from the optic disc to the periphery; a variable amount of retinal haemorrhage, sometimes with the appearance of peripapillary cotton wool spots; an almost invariable absence of vitreous haemorrhage; and remarkably little disturbance of the vision (early or late) except when the macular area is involved in haemorrhage or, when the retinopathy is severe, by a form of cystic maculopathy. Eventually there may be some sheathing of the retinal veins, but this is variable, and the only conspicuous late features are the indefinite persistence of some degree of dilatation of the retinal veins, a postoedematous blurring of the optic disc, and occasionally some disturbance of the macular area.

In a recent comprehensive study of central retinal vasculitis C. D. Hart and colleagues found evidence of a normal retinal arterial pressure (measured by ophthalmodynamometry) and by normal arterial filling (studied by fluorescein angiography), except in one of the nine cases in which there was an associated arterial disease due to malignant hypertension. But there was an obvious abnormality of the retinal veins as shown by the absence of a spontaneous retinal venous pulsation in the affected eye in all cases, by a grossly raised retinal venous pressure in five of the nine cases, and by evidence of a vascular decompensation, which assumed two main patterns. Firstly, a stage of venous decompensation was noted, with dilatation of the retinal veins and leakage of fluorescein dye from the larger veins (perivenous fluorescence). At this stage the retinopathy was mild or moderate-retinal haemorrhages, cotton wool spots, minimal retinal oedema, and more or less normal vision. Secondly, a stage of capillary decompensation, with dilatation of some of the retinal capillaries, often with the formation of microaneurysms, and with extensive leakage of the dye particularly in the macular area. At this stage the retinopathy was severe-considerable retinal oedema and changes in the macular area which resembled those of a cystic maculopathy in addition to the other features of a mild or moderate retinopathy. There were also areas of capillary closure and the appearance of shunt vessels at the optic disc.

It is evident that a reduction in the arteriovenous pressure gradient (perfusion pressure), which is produced solely by a rise in the venous pressure, is a characteristic feature of central retinal vasculitis. It results in a rise in tissue pressure in the retina, which accounts for the areas of capillary closure. It probably also causes an ischaemic cellular oedema and a failure of the tissue fluid to be absorbed by the venous capillaries, since the intraluminal pressure exceeds the plasma protein osmotic pressure. The severity of the retinopathy is almost certainly determined by the site of the lesion in the central retinal vein. When the lesion is proximal to the optic disc the retinopathy is mild-that is, it assumes the form of a venous decompensation-but when it is within the optic nerve or optic nerve-head the retinopathy is much more severe-that is, it assumes the form of a capillary decompensation.
Hart and his colleagues suggest that central retinal vasculitis represents a form of central retinal vein thrombosis in association with an otherwise normal vascular system, so that there is a potential for the development of collateral channels. Hence the retinal changes are less severe and the visual prognosis is better than in the usual forms of central retinal vein thrombosis. This is an interesting concept, and thrombosis almost certainly is an element of some of the more severe cases. But certain points cast doubt on such an emphatic view. An example is the occurrence of central retinal vasculitis in one eye and of peripheral retinal vasculitis (Eales's disease), which is certainly not the result of a thrombotic state, in the other eye. Again, a massive increase in the retinal haemorrhages may follow the use of anticoagulants, yet they may respond favourably in many cases to systemic steroids. ${ }^{3}$ A response to steroids was not experienced by Hart and colleagues, ${ }^{4}$ who favour the use of acetazolamide in an attempt to improve the ocular perfusion.

Certainly the differential diagnosis of central retinal vasculitis is more or less limited to central retinal vein thrombosis, if it is accepted that the two conditions are not identical. As a general rule the retinal haemorrhages in a central retinal vasculitis are less than would be expected in a central retinal vein thrombosis in view of the extent of the disc oedema and the massive retinal vein dilatation which are features of both conditions. Other conditions may be mentioned but they should not cause confusion: plerocephalic oedema is bilateral (as a general rule) and the dilatation of the retinal veins is confined to an area round the optic disc; papillitis is characterized by a distinct loss of vision in the acute stage; and a fulminating hypertensive retinopathy is bilateral and shows other obvious features of widespread arterial disease.

1 Fales, H., Birmingham Medical Review, 1880, 9, 262.

Ballantvne, A. J., and Michaelson, I. C., British fournal of Ophthalmology, 1937, 21, 22.

3 Lvle, T. K., and Wybar, K., British fournal of Ophthalmology, 1961, 45, 778.

4 Hart. C. D., Sanders, M. D., and Miller, S. J. H., British Fournal of Ophthalmology, 1971, 55, 721 .

\section{Food and Cyanide}

In some regions of the world diseases which are relatively uncommon or unknown elsewhere are frequently encountered. There may be many different causes for this, including racial and genetic factors as well as infective agents. If all of these can be safely excluded, attention naturally turns to the food and drink. At times the food incriminated may be esoteric in the extreme. The eating of close relatives' brains in New Guinea, for example, led to the transmission of the fatal disease of kuru. At other times the food may be commonplace, as in some recently investigated tropical neuropathies.

For many years an ataxic neuropathy has been observed in the tropics unlike that due to the common vitamin deficiencies. The patients have a loss of joint position sense leading to a sensory ataxia. This has been ascribed to a posterior column myelopathy, though few necropsies are available. ${ }^{12}$ There is a relatively slight loss of superficial sensation or impairment of lower motor neurons. The sphincters are only rarely affected, and it is uncommon for there to be much impairment of upper motor neurons. The 